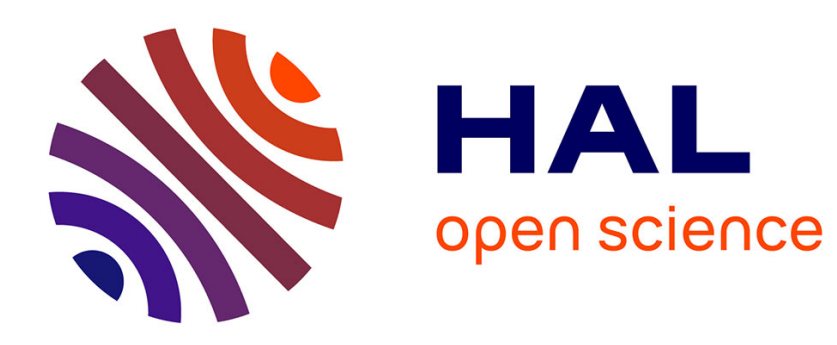

\title{
Étude du phénomène d'échos de spins électroniques : application à la compression d'impulsion radar
}

\author{
Ch. Sourdille
}

\section{To cite this version:}

Ch. Sourdille. Étude du phénomène d'échos de spins électroniques: application à la compression d'impulsion radar. Revue de Physique Appliquée, 1968, 3 (1), pp.73-77. 10.1051/rphysap:019680030107300 . jpa-00242826

\section{HAL Id: jpa-00242826 https://hal.science/jpa-00242826}

Submitted on 1 Jan 1968

HAL is a multi-disciplinary open access archive for the deposit and dissemination of scientific research documents, whether they are published or not. The documents may come from teaching and research institutions in France or abroad, or from public or private research centers.
L'archive ouverte pluridisciplinaire HAL, est destinée au dépôt et à la diffusion de documents scientifiques de niveau recherche, publiés ou non, émanant des établissements d'enseignement et de recherche français ou étrangers, des laboratoires publics ou privés. 


\title{
ÉTUDE DU PHÉNOMÈNE D’ÉGHOS DE SPINS ÉLEGTRONIQUES : APPLIGATION A LA GOMPRESSION D'IMPULSION RADAR
}

\author{
Par Gh. SOURDILLE, \\ Compagnie Française Thomson-Houston $\left.{ }^{(}{ }^{1}\right)$. \\ (Reçu le 8 août 196\%.)
}

\begin{abstract}
Résumé. - Un spectromètre à échos de spins électroniques a été réalisé à $3000 \mathrm{MHz}$. Il a permis de mesurer les temps de relaxations de plusieurs cristaux paramagnétiques, dans un domaine de température variant de $300 \mathrm{oK}$ à $2 \mathrm{oK}$. Parmi ceux-ci, le sodium en solution dans l'ammoniac liquide a été choisi pour des expériences de compression d'impulsion radar. En effet, une modulation de fréquence durant les impulsions permet de réaliser une compression d'impulsion en utilisant le phénomène de "mémoire " des échos de spins.

Les filtres adaptés ou corrélateurs classiques sont ici remplacés par un échantillon paramagnétique situé dans un champ continu inhomogène.

Les limites théoriques et expérimentales du phénomène sont étudiées.

Abstract. - An electron spin-echoe $\mathrm{S}$ band spectrometer has been designed and operated in our laboratory. Some paramagnetic substances were tested in the $300 \mathrm{oK}-2 \mathrm{oK}$ temperature range. Among these, we chose sodium-ammonia solutions for radar pulse compression experiments.

For classical matched filters we substitute a paramagnetic samp'e placed in an inhomogeneous $\mathrm{DC}$ magnetic field.

Theoretical and experimental limits of the phenomenon are given.
\end{abstract}

I. Introduction. - La portée des radars classiques est fonction directe de l'énergie contenue dans l'impulsion émise, alors que le pouvoir discriminateur et la précision en distance sont inversement proportionnels à la durée de cette impulsion. Ces deux impératifs sont contradictoires : une bonne précision impose des impulsions très courtes; l'énergie émise est alors limitée par la puissance crête admissible dans les composants du système d'émission.

Klauder [1], en 1960, propose un système de compression d'impulsion qui permet de se libérer de ces conditions incompatibles en utilisant des filtres adaptés ou corrélateurs.

Mims [2], en 1963, pense à remplacer les appareillages classiques par un corps paramagnétique placé dans un champ continu inhomogène; la compression d'impulsion est alors réalisée par phénomènes d'échos de spins électroniques, dont le principe a été défini par Hahn [3] dans le domaine de la R.M.N.

II. Explication physique du phénomène (fig. 1). L'échantillon paramagnétique est soumis durant toute l'expérience à un champ magnétique continu $H_{0}$

(1) Cette étude a été réalisée aux Laboratoires de Recherches Générales de la C.F.T.H.H.B. dans le cadre d'un marché D.R.M.E.

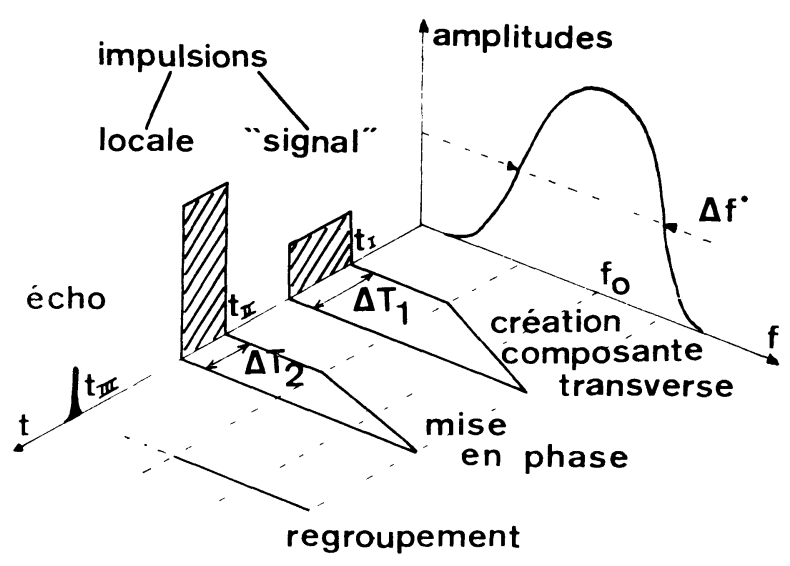

FIG. 1.

Schéma de principe de la compression d'impulsions.

relié à la fréquence de travail $f_{0}$ par l'équation de résonance :

$2 \pi f_{0}=\gamma H_{0} \quad \gamma:$ facteur gyromagnétique.

- L'impulsion radar est matérialisée par une impulsion bas niveau (largeur $\Delta T_{\mathrm{I}}$, modulée linéairement en fréquence sur un domaine $\Delta f$ ) appliquée au temps $t_{\mathrm{I}}$. Durant cette impulsion, les spins d'une raie 
homogène sont basculés quand la fréquence instantanée dans l'impulsion correspond à leur fréquence propre de résonance.

Si la plage de balayage $\Delta f$ est supérieure à la largeur de raie inhomogène $\Delta f^{*}=\gamma \Delta H^{*} / 2 \pi$ (cas d'une raie lorentzienne) $\left(\Delta H^{*}\right.$ : largeur de raie inhomogène en champ magnétique), tous les paquets de spins homogènes seront successivement basculés : on aura créé une composante d'aimantation transverse correspondant à chaque paquet homogène. Cette première impulsion est appelée impulsion « signal ».

- Dès que les spins ont été basculés, ils ne sont plus soumis qu'aux phénomènes de relaxations spins-spins et spins-réseau. Ils vont donc se déphaser de façon cohérente durant un temps $T_{2}^{*}$ (le temps de cohérence de phase), et de façon irréversible et incohérente en un temps $T_{2}$ (temps de mémoire de phase), le retour à l'équilibre thermodynamique s'effectuant en un temps $T_{1}$ (temps de relaxation spin-réseau). Ces trois temps sont liés par l'inégalité :

$$
T_{2}^{*} \leqslant T_{2} \leqslant T_{1} \text {. }
$$

- Sans attendre que tous les spins aient été déphasés de façon irréversible, appliquons au temps $t_{\text {II }}$ une seconde impulsion :

$$
\left(t_{\mathrm{II}}-t_{\mathrm{I}}<T_{2}, T_{1}\right)
$$

Cette impulsion a un niveau tel qu'elle crée sur le système de spins un passage adiabatique qui va faire tourner chaque composante élémentaire d'aimantation de $180^{\circ}$. L'action de cette impulsion, appelée impulsion locale, est analogue à celle créée par une impulsion $\pi$ dans les expériences d'échos de spins : elle remet chaque composante élémentaire d'aimantation en phase.

- Après cette impulsion, les spins ne sont plus soumis qu'aux phénomènes de relaxation; sous l'action de la relaxation spin-spin inhomogène, ils vont se regrouper et induire au temps $t_{\mathrm{III}}\left(t_{\mathrm{III}}-t_{\mathrm{II}}=t_{\mathrm{II}}-t_{\mathrm{I}}\right)$ un signal de résonance paramagnétique, ou « écho de spins ", de largeur $\delta T \sim 1,4 T_{2}^{*}$.

On a donc comprimé l'impulsion « signal » de largeur $\Delta T_{\mathrm{I}}$ en une impulsion de largeur $\delta T$ par l'intermédiaire d'un matériau paramagnétique et d'un signal de remise en forme (impulsion locale).

III. Etudes antérieures et thème orienteur. - L L'étude de Mims [2] a porté sur des cristaux de silicium dopé au phosphore (densité $1,5 \times 10^{17} \mathrm{spins} / \mathrm{cm}^{3}$ ) et de tungstate de calcium dopé au cérium (densité $6 \times 10^{18} \mathrm{spins} / \mathrm{cm}^{3}$ ) à la température de l'hélium liquide. Ces cristaux ont permis d'obtenir des taux de compression $\Delta T_{\mathrm{I}} / \delta T$ de 60 pour des largeurs de bande de l'ordre de $3 \mathrm{MHz}$.

Notre travail a consisté à chercher un cristal utilisable à la température ambiante et à améliorer la bande passante. Nous avons donc mis au point, pour mesurer les temps de relaxations de différents cristaux, un spectromètre à échos de spins; cette réalisation était d'ailleurs une étape logique pour aboutir à un banc de compression d'impulsions.
IV. Spectromètre de résonance électronique par échos de spins. - Le phénomène d'échos de spins se caractérise par l'observation d'un signal de résonance paramagnétique en dehors de tout champ hyperfréquence appliqué; son intérêt est de permettre des mesures des temps de relaxation spin-réseau $T_{1}$ et de spin-spin $T_{\mathbf{2}}$ (temps de mémoire de phase) malgré la présence de fortes homogénéités de champ.

Du fait des temps de relaxation courts en résonance électronique, les conditions à satisfaire pour mettre au point un banc d'échos de spins conduisent à un matériel complexe.

Nous nous étions fixé comme limite des temps de relaxation à mesurer $T_{2}^{*}=50 \mathrm{~ns} \quad\left(T_{2}^{*}\right.$ : temps de cohérence de phase), ceci est équivalent à une largeur de raie inhomogène $\Delta H^{*}$ de $2 \mathrm{E}$. Le coefficient de surtension maximum de la cavité et la largeur de bande minimum du récepteur compatibles avec des temps de 50 ns étaient : $Q_{\mathrm{L}} \sim 150$ (à $3000 \mathrm{MHz}$ ) et $\Delta f \sim 20 \mathrm{MHz}$.

D'autre part, le champ hyperfréquence durant l'impulsion devait être supérieur à la largeur de raie déjà définie pour combattre les phénomènes de relaxation; cette condition imposait une puissance crête, compte tenu du coefficient de surtension faible, supérieure à $5 \mathrm{~W}$. Par contre, en dehors des impulsions, le système paramagnétique ne devait pas être excité, soit : $H_{\mathrm{t} \text { résiduel }} \ll \frac{1}{\gamma \sqrt{T_{1} T_{2}^{*}}} ;$ le calcul montre qu'il faut un découplage entre le sommet de l'impulsion et la résiduelle supérieur à $80 \mathrm{db}$. Enfin, l'introduction d'échantillons variés, à différentes températures, dans la cavité produisait des glissements de fréquence et de couplage; nous y avons remédié en réalisant une cavité à fréquence et couplage variables indépendamment. Un diagramme schématique du spectromètre réalisé à $2960 \mathrm{MHz}$ est donné figure 2 .

Le générateur hyperfréquence délivre $1 \mathrm{~mW}$ de

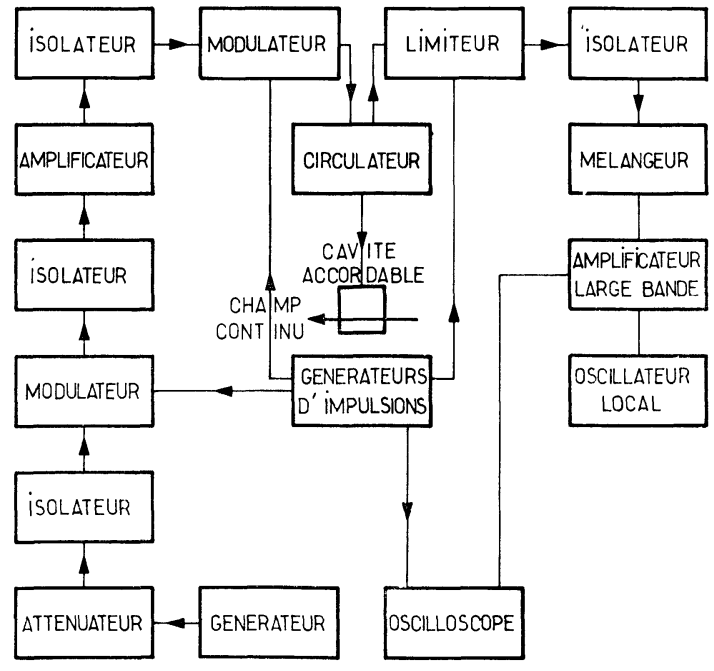

FIG. 2. - Schéma du spectromètre à écho de spins. 
puissance continue stabilisée à $2960 \mathrm{MHz}$. L'onde est ensuite découpée en deux impulsions par un modulateur, constitué par quatre diodes hyperfréquences montées en parallèle à $\lambda g / 4$ dans un guide. (Les caractéristiques de ce modulateur sont : taux de découplage $\geqslant 100 \mathrm{db}$ et des pertes $<0,8 \mathrm{db}$ de 2930 à $3000 \mathrm{MHz}$, les temps de montée et descente sont inférieurs à 15 ns.) Les impulsions hyperfréquences bas niveau sont ensuite amplifiées par un tube à onde progressive et portées à $10 \mathrm{~W}$ crête. Un second modulateur à diode de $50 \mathrm{db}$ de découplage élimine le bruit introduit par le T.O.P.

Les impulsions amplifiées sont transmises par l'intermédiaire d'un circulateur, à une transition guide coaxial, puis à une cavité coaxiale $\lambda / 4$ résonnant sur le mode T.E.M. Le système de couplage par ondes évanescentes permet de régler le coefficient de couplage par translation $\mathrm{du}$ conducteur central. Le réglage en fréquence s'effectue par vissage et dévissage de la tige $\lambda / 4$ de la cavité. La figure 3 donne une vue

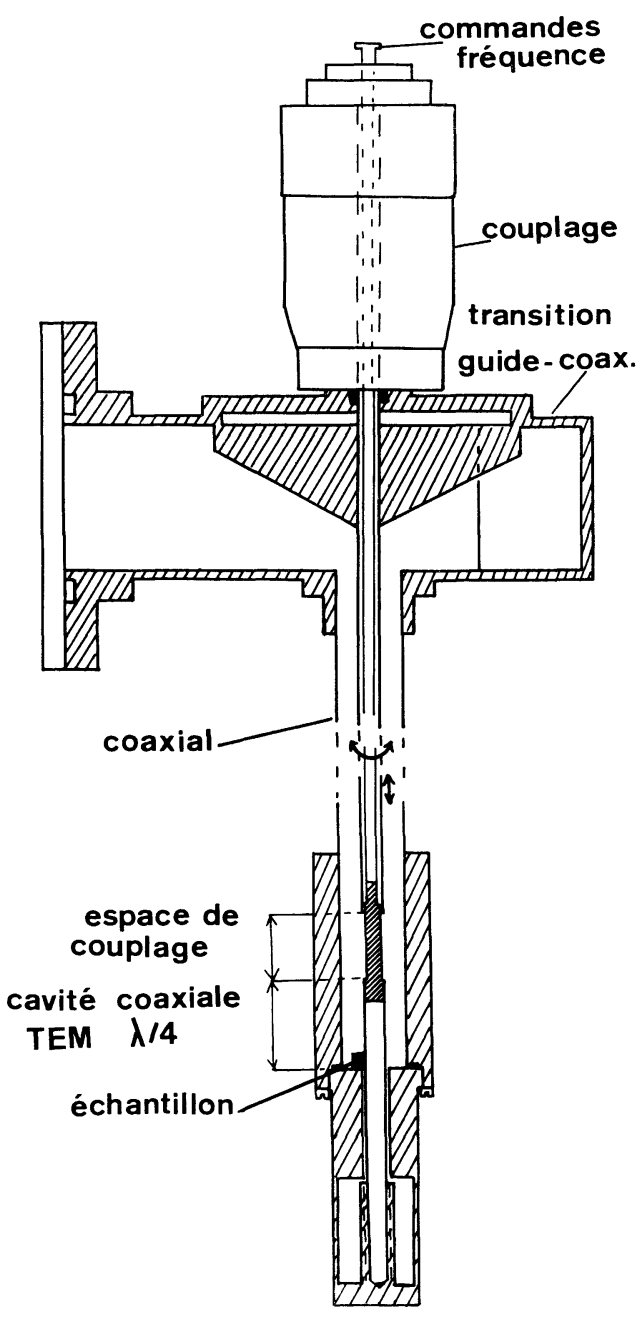

FIG. 3.

Ensemble transition guide coaxial et cavité. d'ensemble de la réalisation transition-cavité. Un limiteur à diode permet d'atténuer le signal réfléchi par la cavité durant les impulsions, on évite ainsi l'éblouissement du récepteur qui empêcherait la détection des échos paramagnétiques dont le niveau se situe en moyenne à $60 \mathrm{db}$ en dessous de la puissance des impulsions hyperfréquences. Le récepteur superhétérodyne a une sensibilité de $10^{-12} \mathrm{~W}$ et une largeur de bande de $40 \mathrm{MHz}$ pour une fréquence intermédiaire de $70 \mathrm{MHz}$. Ce banc présente l'avantage d'être d'utilisation très souple :

- Une modulation de fréquence pendant les impulsions permet de l'utiliser pour les expériences de compression d'impulsion.

- La suppression des circuits d'impulsions le transforme en un banc classique de R.P.E. en ondes entretenues.

- Pour les expériences d'échos de spins, les largeurs des deux impulsions hyperfréquences et du retard entre ces impulsions sont indépendamment variables.

V. Matériau paramagnétique. - Parmi les mesures réalisées avec ce banc en R.P.E. en régime permanent ou transitoire (échos de spins), nous avons sélectionné

\begin{tabular}{|c|c|c|c|c|c|c|}
\hline corps & $\begin{array}{r}\text { spins } \\
/ \mathrm{cm}^{3}\end{array}$ & \begin{tabular}{c|}
$\mathbf{T}$ \\
${ }^{\circ} \mathbf{K}$
\end{tabular} & $\begin{array}{l}\text { largeur } \\
\Delta \mathrm{H}^{\circ} \text { Oe }\end{array}$ & $\begin{array}{l}\text { le raie } \\
T_{2}{ }^{n s}\end{array}$ & $\begin{array}{c}T_{1} \\
\text { ns }\end{array}$ & $\begin{array}{c}\mathrm{T}_{2} \\
\mathrm{~ns}\end{array}$ \\
\hline DPPH & $\begin{array}{c}1,5 \times 10^{21} \\
10^{19}\end{array}$ & \begin{tabular}{|l|}
300 \\
300
\end{tabular} & $\begin{array}{c}1,84 \\
14\end{array}$ & $\begin{array}{c}62 \\
8\end{array}$ & 80 & 80 \\
\hline SilP & $\begin{array}{l}1,5 \times 10^{17} \\
6 \times 10^{17}\end{array}$ & $\begin{array}{l}4,2 \\
4,2\end{array}$ & $\overbrace{14}^{z_{4} \int_{4} \int_{40}^{2}}$ & 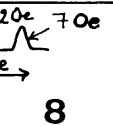 & & \\
\hline $\begin{array}{c}(\mathrm{Ca}, \mathrm{Ce}) \\
\mathrm{WO}_{4}\end{array}$ & $2,5 \times 10^{19}$ & 4,2 & $\min .7,75$ & & & \\
\hline $\begin{array}{l}(\mathrm{La}, \mathrm{Ce}) \\
\mathrm{NO}_{3}\end{array}$ & $3 \times 10^{18}$ & 2 & 3,75 & 30 & & \\
\hline $\mathrm{Na} / \mathrm{NH}_{3}$ & $10^{20}$ & 300 & 0,48 & 220 & $3.10^{3}$ & $3.10^{3}$ \\
\hline $\begin{array}{l}\text { phényl } \\
\text { allyl }\end{array}$ & $10^{21}$ & $\begin{array}{c}300 \\
77\end{array}$ & $\begin{array}{c}0,6 \\
1\end{array}$ & $\begin{array}{l}190 \\
110\end{array}$ & & $\begin{array}{l}190 \\
110\end{array}$ \\
\hline
\end{tabular}

FIG. 4.

Caractéristiques des corps paramagnétiques mesurés.

les plus caractéristiques pour dresser le tableau de la figure 4. Les matériaux utilisés étaient les suivants :

- Radicaux libres : D.P.P.H., $\alpha \beta$ bidisphénylène $\gamma$ phénylallyl;

- Terres rares : tungstate de calcium dopé au cérium, nitrate double de lanthane et de magnésium dopé au cérium; 
- Métaux alcalins : sodium en solution dans l'ammoniac liquide;

- Semi-conducteur : silicium dopé au phosphore.

Ces mesures nous ont permis de déterminer le temps de résolution de notre appareillage qui est de l'ordre de $80 \mathrm{~ns}$. Le tableau de la figure 4 montre que seul le sodium en solution dans l'ammoniac à des temps de relaxations supérieurs à la microsonde et donc compatibles avec des expériences de compression d'impulsion.

Nous avons effectué sur ce corps des mesures systématiques de temps de relaxation en fonction de la concentration et de la température. $T_{2}$ a été mesuré par méthode de doubles impulsions (fig. 5) et $T_{1}$ par méthode d'impulsions triples.

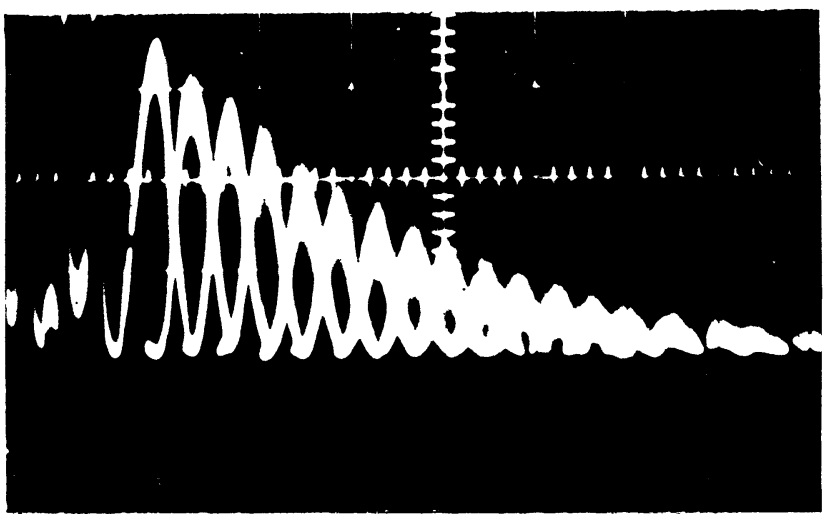

FIG. 5.

Mesure de $\mathrm{T}_{2}$ : impulsions doubles (800 ns/div.).

Nous avons trouvé $T_{1}=T_{2}$ dans tous les cas, ce qui correspond bien à la théorie [4], [5], $T_{2}^{*}$ variait

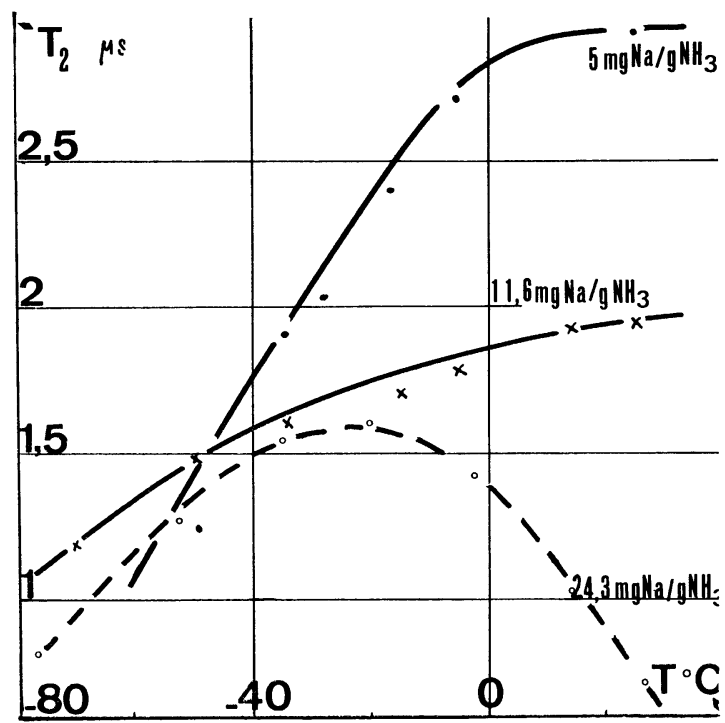

FIG. 6. - Variation des temps de relaxation en fonction de la température et de la concentration (sodium en solution dans l'ammoniac). de 300 ns à 80 ns suivant l'homogénéité du champ extérieur appliqué. La figure 6 résume les résultats obtenus pour les concentrations extrêmes et la concentration centrale des échantillons approvisionnés.

VI. Compression d'impulsion. - D'après les résultats de la figure 6 , nous avons choisi une concentration de $5 \mathrm{mg}$ de sodium par gramme d'ammoniac à $20^{\circ} \mathrm{C}$ $\left(\sim 10^{20}\right.$ spins $\left./ \mathrm{cm}^{3}\right)$; les temps de relaxation de l'ordre de $3 \mu$ s ont permis de réaliser des expériences de compression d'impulsion. Pour expérimenter et mettre en évidence le phénomène de compression d'impulsion, il a fallu modifier légèrement le banc de la figure 2. Nous avons vu qu'il fallait effectuer une modulation de fréquence linéaire durant les impulsions; cette condition est réalisée en appliquant à l'hélice d'un carcinotron deux dents de scie synchronisées aux impulsions. D'autre part, l'impulsion « signal » était maintenue à faible niveau par blocage du tube à onde progressive au moyen d'une forte impulsion négative appliquée sur la grille du tube durant le temps de passage de l'impulsion « signal ».

Nous avons vérifié que pour des réglages tels que :

$$
\Delta T_{\mathrm{I}}=2 \Delta T_{\mathrm{II}}<T_{2} \quad \text { et } \quad \Delta f>\Delta f^{*}
$$

nous obtenions une impulsion comprimée de largeur $1,4 T_{2}^{*}<T_{2}$.

Les caractéristiques du système sont :

- Le taux de compression $\frac{\Delta T_{\mathrm{I}}}{\delta T} \leqslant \frac{T_{2}}{T_{2}^{*}}$;

- L'efficacité, dont nous avons calculé la valeur théorique en bonne concordance avec l'expérience [7]:

$\frac{P_{\mathrm{e}}}{P_{\mathrm{I}}}=\frac{(2 \beta)^{2}}{(1+\beta)} \frac{\gamma^{4} h^{4}}{16 \mu_{0}^{2} k^{2}} \frac{N^{2}}{T^{2}} \frac{\left(V_{0}\right)^{2}}{\left(V_{\mathrm{c}}\right)} \frac{\omega_{0}^{4}}{\pi^{2}} \frac{\Delta T_{\mathrm{I}}}{\left(\Delta f^{*}\right)^{3}}$

(en MKSA)

$P_{\mathrm{e}}$ : puissance de l'impulsion comprimée,

$P_{\mathrm{I}}$ : puissance de l'impulsion « signal »,

$h$ : constante de Planck,

$k$ : constante de Boltzmann,

$V_{\mathrm{s}}$ : volume de l'échantillon,

$V_{\mathrm{c}}$ : volume de la cavité,

$N$ : densité de spins.

On a donc intérêt à augmenter fortement le volume de l'échantillon et à élargir dans les limites de $T_{2}$, l'impulsion signal, pour augmenter l'efficacité. Par contre, le fait d'augmenter la bande passante (augmentation de l'inhomogénéité extérieure) augmente le taux de compression, mais diminue cette efficacité.

Le meilleur taux de compression obtenu a été de 30 avec une largeur de bande de $15 \mathrm{MHz}$ (largeur d'impulsion comprimée $: \sim 80 \mathrm{~ns}$ ) ( fig. 7).

L'efficacité du système était de $10^{-7}$ avec un rapport 
signal sur bruit de $22 \mathrm{db}$, tous ces résultats étant en bon accord avec la théorie.

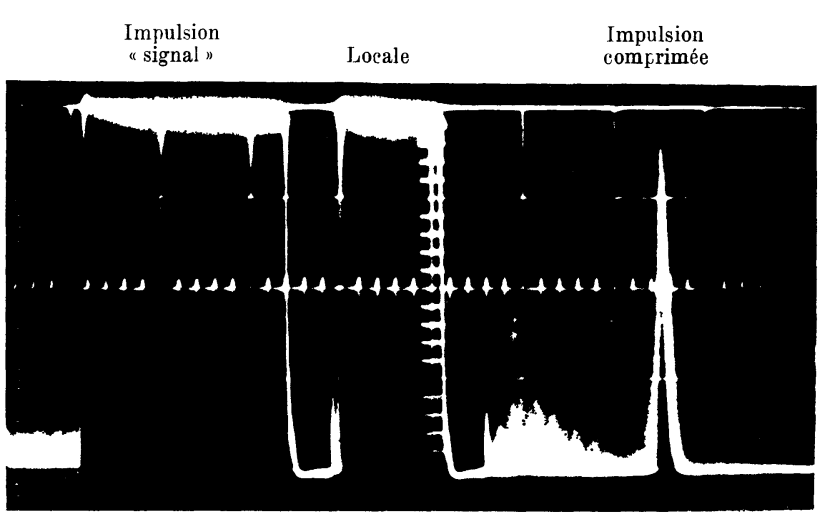

Fig. 7. - Compression d'impulsion (1 $\mu \mathrm{s} /$ div.).
Conclusion. - Cette étude a permis de réaliser un spectromètre à échos de spins. Son efficacité pourrait être améliorée d'un facteur 30 par une réalisation en bande $\mathrm{X}$; la gamme des matériaux à temps de relaxation mesurables pourrait être élargie par usage d'un amplificateur à T.O.P. de puissance de saturation supérieure à $100 \mathrm{~W}$. Des mesures systématiques des temps de relaxation $T_{1}$ et $T_{2}$ sur différents cristaux avec un tel spectromètre devraient permettre de trouver un cristal s'adaptant mieux aux conditions de compression d'impulsion et d'augmenter ainsi le taux de compression et l'efficacité du système (forte concentration, faibles pertes diélectriques pour permettre un bon coefficient de remplissage, temps de relaxation longs).

Manuscrit reçu le 8 août 1967.

\section{BIBLIOGRAPHIE}

[1] KLAUDER, The theory and design of chirp-radar, Bell syst. tech. J., 1960, 34, 745.

[2] Mims, Detection of chirped radar signals by electron spin echoes, P.I.E.E.E., 1963, 51, 1130.

[3] Hahn, Spin echoes, Phys. Rev., 1950, 30, 580.

[4] BLUME, Electron spin relaxation times in sodium ammonia solutions, Phys. Rev., 1958, 109, 1867.
[5] Polifak, Electron spin relaxation in metal ammonia solutions, J. Chem. Physics, 1961, 34, 864.

[6] Grangeon (J.), Compression d'impulsion radar au moyen du phénomène d'échos de spins, Rapport interne C.F.T.H.H.B., 1964.

[7] GrangeOn (J.), Sourdille (C.), Étude de la compression d'impulsions par utilisation du phénomène d'échos de spins, Rapport D.R.M.E., décembre 1966 . 